\title{
A Model to Describe the Magnetomechanical Behavior of Martensite in Magnetic Shape Memory Alloy
}

\author{
Zaoyang Guo, ${ }^{1,2}$ Haitao Li, ${ }^{1,2}$ Qiang Wan, ${ }^{3}$ Xianghe Peng, ${ }^{1,2}$ and Jing Wen ${ }^{1,2}$ \\ ${ }^{1}$ College of Aerospace Engineering, Chongqing University, Chongqing 400044, China \\ ${ }^{2}$ Chongqing Key Laboratory of Heterogeneous Material Mechanics, Chongqing University, Chongqing 400044, China \\ ${ }^{3}$ China Academy of Engineering Physics, Mianyang 621900, China
}

Correspondence should be addressed to Haitao Li; htli@cqu.edu.cn

Received 28 November 2013; Accepted 5 June 2014; Published 17 June 2014

Academic Editor: Daniel Balint

Copyright (C) 2014 Zaoyang Guo et al. This is an open access article distributed under the Creative Commons Attribution License, which permits unrestricted use, distribution, and reproduction in any medium, provided the original work is properly cited.

\begin{abstract}
A model to describe the constitutive behavior of magnetic shape memory alloy composed with pure martensite is proposed based on the analysis of variants reorientation. A hyperbolic tangent expression is given to describe the variants transition during magnetic and mechanical loading process. The main features of magnetic shape memory alloy, such as pseudoelastic and partially pseudoelastic behavior as well as minor hysteretic loops, can be successfully replicated with the proposed model. A good agreement is achieved between calculated results and experimental data for NiMnGa single crystal.
\end{abstract}

\section{Introduction}

Magnetic shape memory alloy is one type of intelligent materials, which combines the properties of ferromagnetism with shape memory effect. It can transform magnetic or thermal energy into mechanical response, which produces the actuation. Since Ullakko et al. [1] observed a magneticfield-induced strain of $0.2 \%$ in 1996, large field-induced strain of $9.5 \%$ has been achieved in Ni-Mn-Ga single crystal [2], which is much greater than those of magnetostrictive, piezoelectric, or electrostrictive. In addition, the material has a wide bandwidth of strain response, reported to be within $\mathrm{kHz}$ range [3]. In contrast, the thermally driven shape memory alloy has a slow response of less than $1 \mathrm{~Hz}$. Hence magnetic shape memory alloys can be used as actuators and other pieces of equipment requiring high dynamic response.

The behaviors of magnetic shape memory alloys, such as magnetic-field-induced strain, pseudoelastic response, and other properties, have been studied experimentally in the literature. Kainuma et al. [4] investigated the shape memory and magnetic properties of a NiCoMnSn Heusler polycrystalline alloy. Krenke et al. [5] studied the magnetic superelasticity and inverse magnetocaloric effect in NiMnIn alloy. Dong et al. [6] explored the effect of isothermal ageing on the martensitic transformation and mechanical properties of NiMnGaTi alloy.

In addition to experimental investigations, it is also important to predict the behaviors of magnetic shape memory alloys for their applications. Researchers have proposed some constitutive models associated with the martensitic variant reorientation [7-16]. Most of these models are based on the minimization of a free energy function. O'Handley et al. [7] proposed a free energy expression including Zeeman energy, magnetic anisotropy energy, and internal elastic energy, and the field and stress dependence of the strain can be accounted for by minimization of this energy. Liang et al. [10] developed a thermodynamic model where Gibbs free energy is a function of temperature and magnetic field to study the phenomena of lowering the transformation temperature by a magnetic field. Glavatska et al. [11] suggested a statistical model of the partially reversible magnetostrain effect in the twinned thermoelastic martensite using Gibbs potential of a cubic crystal. Zhu and Dui [13] used a thermodynamic method for martensite transformation criterion based on the analysis of Gibbs free energy, accounting for the influence of variants morphology and material properties. Wang and $\mathrm{Li}$ [15] proposed a kinetics model describing macroscopic behavior of martensitic variants rearrangement 
based on a tensor description of thermodynamic continuum mechanics considering magnetomechanical coupling on the rearrangement process. Kiefer et al. [16] presented the constitutive models for forward and reverse reorientation and corresponding Gibbs free energy. The evolution of crystallographic structure can be captured through the evolution of internal state variables. In the above models, some of them have many parameters to be determined, which is due to the complex expression for Gibbs free energy, inducing inconvenient for application. For example, in L'vov's model [12], the Gibbs potential includes Helmholtz free energy, free energy of magnetic subsystem of the crystal, the energy of magnetoelastic coupling, and mechanical energy, which makes the calibration of model parameters very difficult.

In this paper, a simple phenomenological model is proposed to describe the magnetomechanical behaviors of magnetic shape memory alloys in martensite. The theoretical predictions from this model agree well with the experimental results.

\section{Theoretical Model Based on Variants Reorientation}

To understand the mechanism of variants reorientation during loading process, a schematic for microstructure evolution is shown in Figure 1. Assuming that magnetic shape memory alloy is initially martensitic and sufficiently large external magnetic field is applied, a single variant martensite can be obtained. The variant is defined as field-preferred variant (variant I) with short axis of lattice along magnetic field direction. When compressive stress perpendicular to short axis is applied, the response of magnetic shape memory alloy is elastic until the critical stress is achieved. Subsequently twin boundary motion and martensite reorientation will occur, and another stress-preferred variant (variant II) with short axis along compression direction will be induced. The volume fraction of variant II will increase with larger compressive stress. In this stage, the stress-strain behavior is nonlinear. When reorientation is finished, the martensite is composed of pure variant II. So the response of magnetic shape memory alloy is actually the behavior of another single variant martensite if the specimen is subjected to further compression.

Up to now, most of experimental investigations focus on the magnetomechanical properties of magnetic shape memory alloy in martensite. For this case, the total deformation can be described as

$$
\boldsymbol{\varepsilon}=\boldsymbol{\varepsilon}^{e}+\boldsymbol{\varepsilon}^{r}
$$

where $\boldsymbol{\varepsilon}^{e}$ is the elastic strain and $\boldsymbol{\varepsilon}^{r}$ denotes the strain for variants reorientation in martensite, which is called magnetic field-induced strain.

The constitutive equation is expressed as

$$
\boldsymbol{\sigma}=\mathbf{D}: \boldsymbol{\varepsilon}^{e}=\mathbf{D}:\left(\boldsymbol{\varepsilon}-\boldsymbol{\varepsilon}^{r}\right),
$$

where $\mathbf{D}$ is the elasticity tensor.

The external mechanical loads or magnetic field may lead to the reorientation from one variant to another, inducing

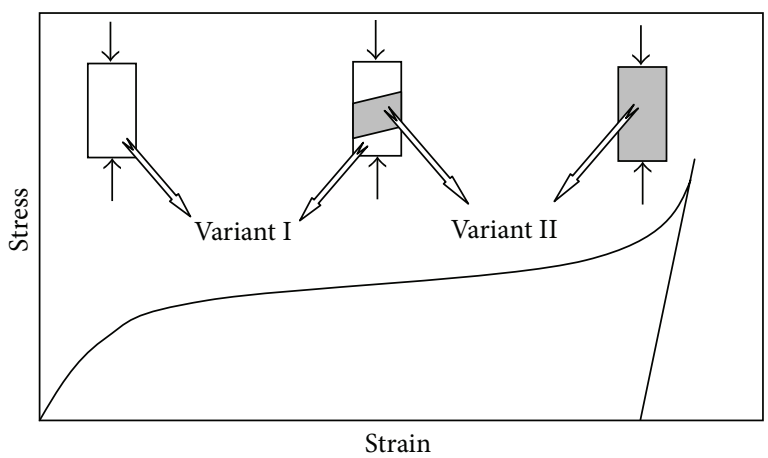

FIGURE 1: A schematic for microstructure evolution and corresponding stress-strain behavior of magnetic shape memory alloy in martensite during the loading process.

reorientation strain $\boldsymbol{\varepsilon}^{r}$. For magnetic shape memory alloy single crystal in martensitic phase, $\boldsymbol{\varepsilon}^{r}$ is related to the volume fraction of variants. Assuming that $\xi_{\sigma}$ represents the volume fraction of stress-preferred variant, the field-preferred variant $\xi_{H}$ is

$$
\xi_{H}=1-\xi_{\sigma}
$$

In one-dimensional condition, the maximum reorientation strain is assumed to be $\varepsilon_{\max }^{r}$; that is, the material is initially composed entirely of stress-preferred variant and finally field-preferred variant, or a reverse transition. In the experiments, the initial strain of the material in a free state is usually set to zero, thus the reorientation strain can be described as

$$
\varepsilon^{r}= \begin{cases}\xi_{H} \varepsilon_{\max }^{r} & \text { initially stress-preferred variant } \\ -\xi_{\sigma} \varepsilon_{\max }^{r} & \text { initially field-preferred variant. }\end{cases}
$$

The one-dimensional constitutive relation then reads

$$
\sigma=E\left(\varepsilon-\varepsilon^{r}\right) \text {. }
$$

It should be noted that Young's modulus $E$ depends on variant fraction based on experimental investigations and can be expressed as a function of stress-preferred variant, which is similar to the equation in shape memory alloys suggested in [18]:

$$
E=E\left(\xi_{\sigma}\right)=E_{H}+\xi_{\sigma}\left(E_{\sigma}-E_{H}\right),
$$

where $E_{H}$ and $E_{\sigma}$ are Young's moduli for martensite with entirely magnetic field-preferred variant and stress-preferred variant, respectively. The experimental evidence shows that $E_{\sigma}$ is usually larger than $E_{H}[14]$.

For shape memory alloys, the most used phenomenal expression to describe the phase transformation has a form of exponential function [19] or cosine function [20]. Unlike these functions, we select a hyperbolic tangent equation to describe the reorientation of martensite variants:

$$
\xi_{\sigma}= \begin{cases}\frac{1}{2}\left\{\tanh \left[K_{s}\left(\sigma-\bar{\sigma}_{s}(H)\right)\right]+1\right\} & V_{M} \longrightarrow V_{S} \\ \frac{1}{2}\left\{\tanh \left[K_{m}\left(\sigma-\bar{\sigma}_{m}(H)\right)\right]+1\right\} & V_{S} \longrightarrow V_{M}\end{cases}
$$


where $V_{M} \rightarrow V_{S}$ represents the reorientation from magnetic field-induced variant to stress-induced variant; $K_{s}$ and $K_{m}$ are material constants related to variants reorientation and can be obtained by fitting the stress-strain curve during uniaxial loading; and $\bar{\sigma}_{s}(H)$ is the average value of $\sigma_{s}^{\mathrm{cr}, s}(H)$, the starting critical stress, and $\sigma_{s}^{\mathrm{cr}, f}(H)$, the stress at the end point of the critical range, during the $V_{M} \rightarrow V_{S}$ process; that is,

$$
\bar{\sigma}_{s}(H)=\frac{1}{2}\left(\sigma_{s}^{\mathrm{cr}, s}(H)+\sigma_{s}^{\mathrm{cr}, f}(H)\right) .
$$

Similarly,

$$
\bar{\sigma}_{m}(H)=\frac{1}{2}\left(\sigma_{m}^{\mathrm{cr}, s}(H)+\sigma_{m}^{\mathrm{cr}, f}(H)\right) .
$$

If the reorientation starts from a state of mixed fieldpreferred variant and stress-preferred variant, (7) can be rewritten as

$$
\xi_{\sigma}= \begin{cases}\xi_{\sigma}^{s}+\frac{1-\xi_{\sigma}^{s}}{2}\left\{\tanh \left[K_{s}\left(\sigma-\bar{\sigma}_{s}(H)\right)\right]+1\right\} & V_{M} \longrightarrow V_{S} \\ \frac{\xi_{\sigma}^{m}}{2}\left\{\tanh \left[K_{m}\left(\sigma-\bar{\sigma}_{m}(H)\right)\right]+1\right\} & V_{S} \longrightarrow V_{M}\end{cases}
$$

where $\xi_{\sigma}^{s}$ and $\xi_{\sigma}^{m}$ are initial fractions during the reorientation in every loading or unloading process.

It is important to note that the average value of critical stress varies with the intensity of magnetic field. Considering the magnetic saturation, the critical stress is nonlinear with the magnetic field and has a saturation value, which is verified by the experimental investigation [17]. Using the concept of magnetostress which is defined as the difference in the stress levels with and without magnetic field [21], the following relation can be obtained:

$$
\bar{\sigma}_{s}(H)=\bar{\sigma}_{s}^{0}+\bar{\sigma}_{s}^{H}(H), \quad \bar{\sigma}_{m}(H)=\bar{\sigma}_{m}^{0}+\bar{\sigma}_{m}^{H}(H),
$$

where $\bar{\sigma}_{s}^{0}, \bar{\sigma}_{m}^{0}$ are mechanical stresses and $\bar{\sigma}_{s}^{H}(H), \bar{\sigma}_{m}^{H}(H)$ are magnetostresses. Generally, $\bar{\sigma}_{s}^{H}(H)=\bar{\sigma}_{m}^{H}(H)$. Taking into account the nonlinear property between magnetostress and magnetic field, the magnetostress can be expressed as

$$
\begin{aligned}
\bar{\sigma}_{s}^{H}(H) & =\bar{\sigma}_{m}^{H}(H)=\bar{\sigma}^{H}(H) \\
& =\frac{1}{2}\left\{\tanh \left[C_{0}\left\langle H-H_{0}\right\rangle\right]+1\right\} \bar{\sigma}_{\text {sat }}^{H}=C(H) \cdot H,
\end{aligned}
$$

where $\bar{\sigma}_{\text {sat }}^{H}$ is the saturation magnetostress, $H_{0}$ is the threshold magnetic field yielding magnetostresses, and $C_{0}$ is a constant and can be determined by fitting the $\bar{\sigma}^{H}-H$ curve:

$$
\left\langle H-H_{0}\right\rangle= \begin{cases}0, & \text { if } H-H_{0} \leq 0 \\ H-H_{0}, & \text { if } H-H_{0}>0,\end{cases}
$$

$C(H)$ is the magnetic field influence coefficient and could be expressed as

$$
C(H)=\frac{\bar{\sigma}_{\text {sat }}^{H}\left\{\tanh \left[C_{0}\left\langle H-H_{0}\right\rangle\right]+1\right\}}{2 H} .
$$

Substituting (12) into (10) yields a new form to describe the fraction of stress-preferred variant:

$$
\xi_{\sigma}=\left\{\begin{array}{c}
\xi_{\sigma}^{s}+\frac{1-\xi_{\sigma}^{s}}{2}\left\{\tanh \left[K_{s}\left(\sigma-\bar{\sigma}_{s}^{0}-C(H) H\right)\right]+1\right\} \\
V_{M} \longrightarrow V_{S} \\
\frac{\xi_{\sigma}^{m}}{2}\left\{\tanh \left[K_{m}\left(\sigma-\bar{\sigma}_{m}^{0}-C(H) H\right)\right]+1\right\} \\
V_{S} \longrightarrow V_{M} .
\end{array}\right.
$$

Using (4), (5), and (15), the mechanical behaviors of the magnetic shape memory alloy under different magnetic field can be described, and the hysteretic loops can also be predicted.

\section{Results and Discussions}

In order to verify the validity of the proposed model, the predictions are compared with experiments carried out by Couch et al. [17]. In the constant field test, a single crystal, martensite, NiMnGa rod was magnetized with high field to ensure the material was initially in field-preferred state. Then it was exposed to a low uniform external magnetic field and the quasi-static compressive stress perpendicularly to the field was applied and increased to $5 \mathrm{MPa}$. Subsequently the load was removed quasi-statically. This test process was cycled under various levels of constant magnetic fields. The parameters used in proposed model are shown in Table 1.

Figure 2 shows the stress-strain curve of magnetic shape memory alloy under external magnetic field of $6 \mathrm{kOe}$. In the loading process, the strain is initially linear with the stress, and the martensite is composed of pure field-preferred variant. So the behavior of NiMnGa is actually the behavior of variant I (see Figure 1). The corresponding stiffness of material $E_{H}$ can be determined. Once the compressive stress reaches a critical value the twin boundary motion is induced. The martensite transforms from field-preferred variant to stress-preferred variant until second critical stress is achieved. The average value of critical stress $\bar{\sigma}_{s}(H)$, material constant $K_{s}$, and maximum reorientation strain $\varepsilon_{\max }^{r}$ can be obtained at this stage. If compression continues to increase the behavior of NiMnGa with variant II is linearly elastic and the stiffness of $E_{\sigma}$ is obtained. In the unloading process, $\bar{\sigma}_{m}$ and $K_{m}$ can also be identified in the reverse transition from variant II to variant I. It can be seen in Figure 2 that the mechanical behavior is pseudoelastic. The prediction of the proposed model shows good agreement with the experimental data.

In order to verify the ability of the model to capture the influence of magnetic field, stress-stain behaviors under different applied magnetic fields are shown in Figure 3. Obviously the moduli of variant I and variant II are unaffected by the magnetic field intensity, but the critical stresses implying the start and end of variant reorientation are affected significantly by the magnetic field. In addition, the hysteretic loops are pseudoelastic at $4 \mathrm{kOe}$ and partially pseudoelastic at $2 \mathrm{kOe}$, which means that the strain does not return to zero upon removal of the load and has a residual value at $2 \mathrm{kOe}$. It can be found that the behavior of material under different fields can be well described by the theoretical model. 
TABLE 1: Material constants measured from experiments [17].

\begin{tabular}{ccccccccccc}
\hline$E_{\delta}(\mathrm{GPa})$ & $E_{H}(\mathrm{GPa})$ & $\varepsilon_{\max }^{r}$ & $K_{s}(\mathrm{MPa})^{-1}$ & $K_{m}(\mathrm{MPa})^{-1}$ & $\bar{\sigma}_{s}^{0}(\mathrm{MPa})$ & $\bar{\sigma}_{m}^{0}(\mathrm{MPa})$ & $C_{0}(\mathrm{kOe})^{-1}$ & $H_{0}(\mathrm{kOe})$ & $\bar{\sigma}_{\text {sat }}^{H}(\mathrm{MPa})$ \\
\hline 9 & 8 & $5.4 \%$ & 2 & 2 & 0.45 & -0.5 & 0.34 & 4.2 & 3 \\
\hline
\end{tabular}

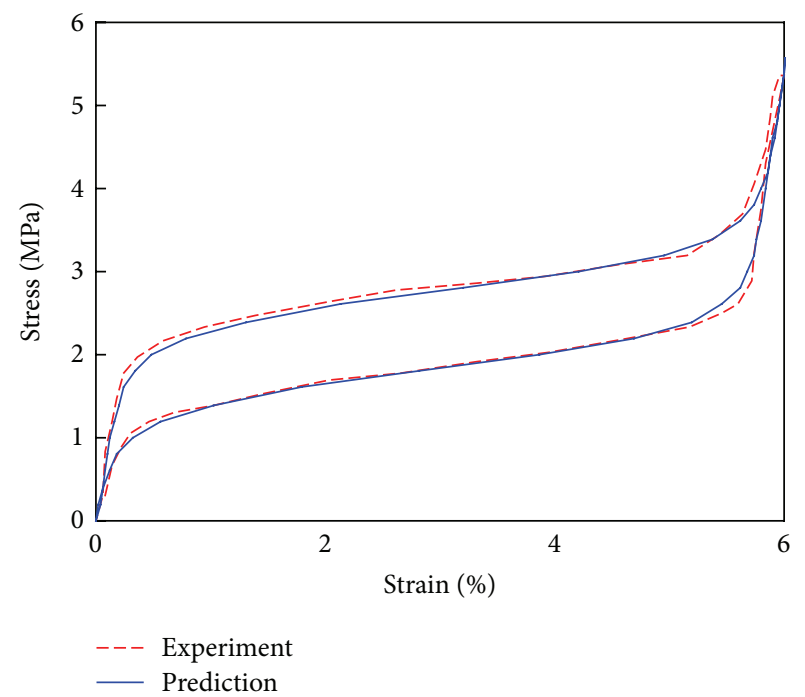

FIgURE 2: Pseudoelasticity of magnetic shape memory alloy in martensite at $6 \mathrm{kOe}$. The prediction is compared with experimental data from [17].

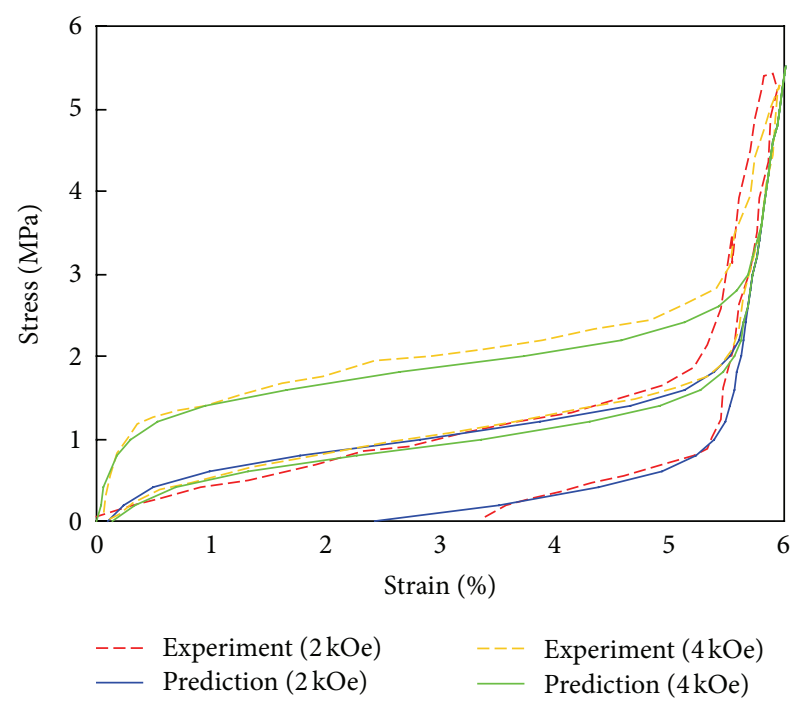

FIGURE 3: Effect of magnetic field on the stress-strain behavior of magnetic shape memory alloy in martensite, which is partially pseudoelastic at $2 \mathrm{kOe}$ and pseudoelastic at $4 \mathrm{kOe}$.

It should be pointed out that the critical stress will reach saturation if magnitude of field intensity is sufficiently large. The proposed model is compared with Couch's model as well as the experimental results at $8 \mathrm{kOe}$ [17] (Figure 4). Couch's quasi-static model fails to capture the smooth transitions between variant I and variant II. It also overpredicted the material stress at higher magnetic field. This limitation

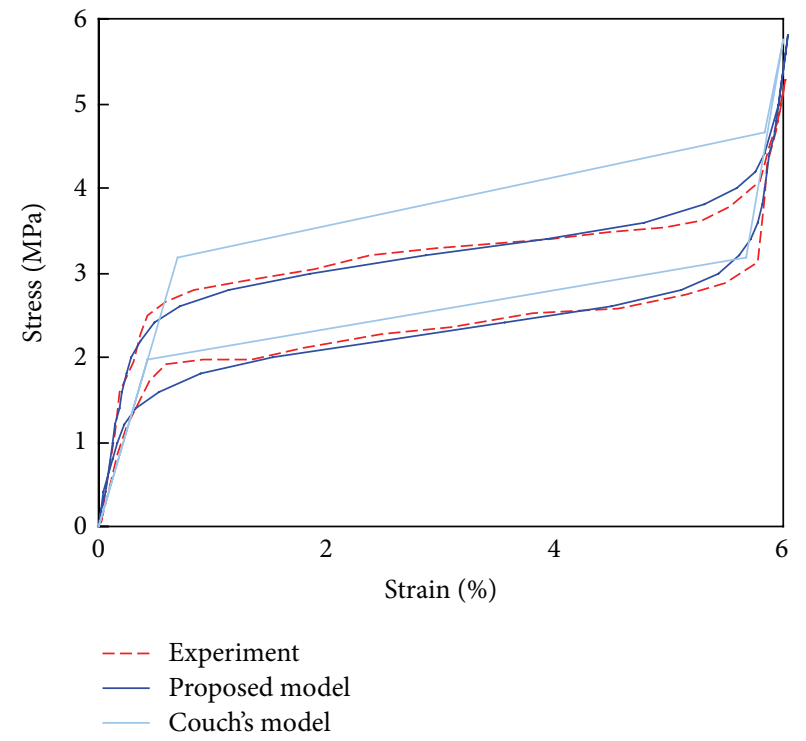

FIgUre 4: Comparison of Couch's quasi-static model and proposed model. The corresponding experiment is carried out at an $8 \mathrm{kOe}$ external magnetic field.

comes from the assumption that the critical stress is linear with applied field. In fact, the relationship between them is complex.

The effect of magnetic field on critical stress can be further analyzed using the definition of magnetostress which is explained in Section 2. The average critical stress $\bar{\sigma}_{s}$ consists of mechanical stress $\bar{\sigma}^{0}$ and magnetostress $\bar{\sigma}^{H}$. Mechanical stress can be determined during the loading process without external magnetic field. Magnetostress can be obtained from the overall stress with different magnetic fields. Figure 5 shows the magnetostress profile for a range of field intensities. It can be seen that $\bar{\sigma}^{H}$ increases with the increase of magnetic field, but the relationship between them is nonlinear. $\bar{\sigma}^{H}$ reaches saturation at large external field. This tendency can be well described by the model.

The hysteretic loops of magnetic shape memory alloy analyzed above imply the absolute transition between fieldpreferred variant and stress-preferred variant. The proposed model can also capture the minor hysteretic behavior of material, which is shown in Figure 6. It must be addressed that the initial fraction of variant should be identified if the variant rearrangement is not accomplished. The calculated result shows a good agreement with experimental data.

\section{Conclusions}

This paper presents a simple model to describe the behavior of magnetic shape memory alloy in martensite. The model 


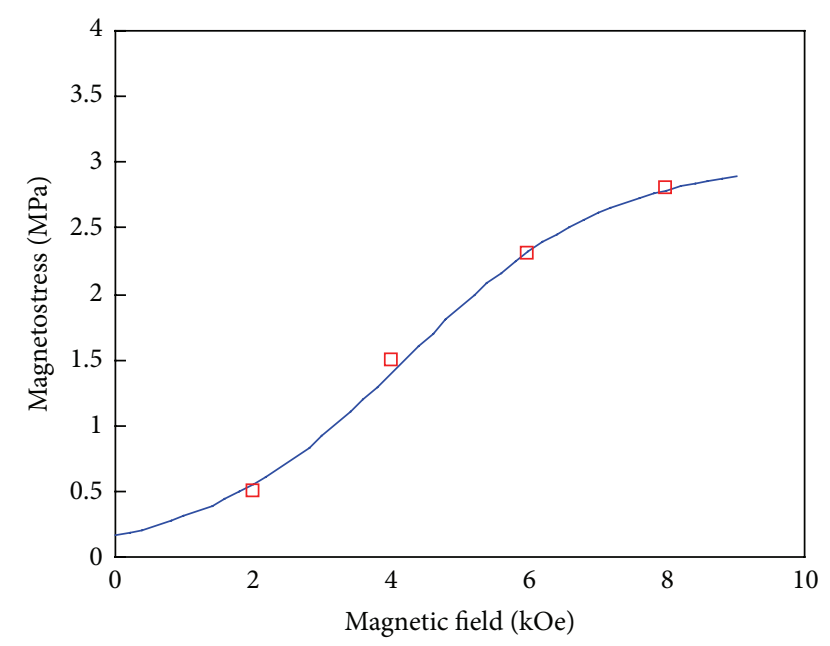

$\square \quad$ Experiment

Prediction

FIGURE 5: Magnetostress in average critical stress when different magnetic field is applied.

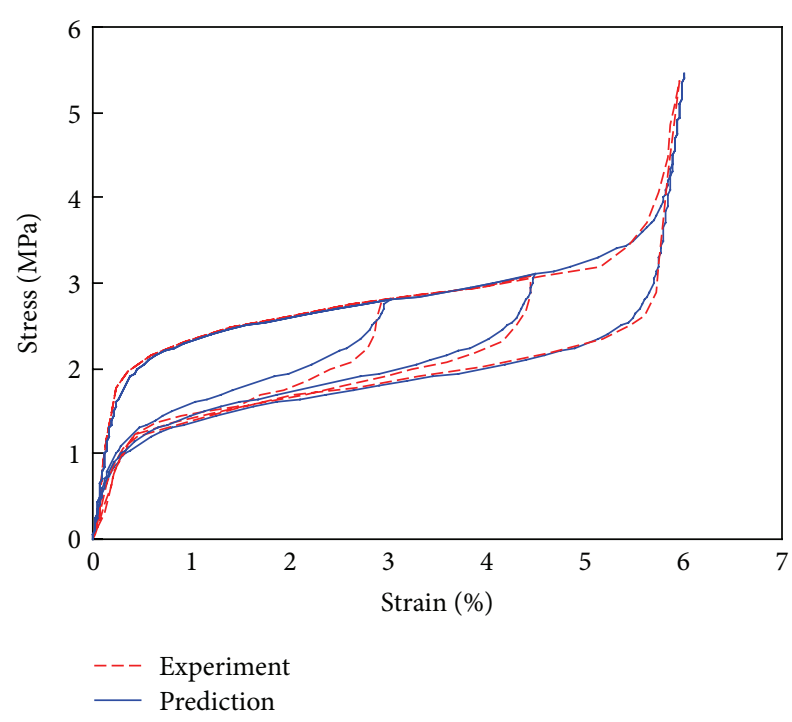

FIGURE 6: Minor hysteretic loops of magnetic shape memory alloy in martensite at $6 \mathrm{kOe}$.

is based on the analysis of microstructure evolution in martensite during the loading process under an external magnetic field. Two types of variants, denoted, respectively, the field-preferred and stress-preferred variant, will transform each other under different conditions. So the material properties, such as stiffness, will be changed during hysteretic loops. Considering the nonlinear character of stress-strain curves involved with variants transition, a hyperbolic tangent equation was proposed to describe the reorientation. In order to investigate the effect of magnetic field on critical stress, the overall stress is decomposed into mechanical stress and magnetostress. Mechanical stress is not affected by the field. Nevertheless, the magnetostress has a remarkable dependence on magnetic intensity and would reach saturation at large field.

The parameters in the proposed model can be determined easily from the stress-strain curves under different magnetic and mechanical loading process. In order to validate the theoretical model, the model predictions are compared with the experimental data and good quantitative agreement is achieved. This model is also compared with Couch's model and it was found that the model proposed here yields more accurate predictions for nonlinear response and higher-order effects such as magnetic saturation. The pseudoelastic and partially pseudoelastic behavior as well as minor hysteretic loops of NiMnGa single crystal in martensite can also be captured by this model.

\section{Conflict of Interests}

The authors declare that there is no conflict of interests regarding the publication of this paper.

\section{Acknowledgment}

The authors are grateful for financial support from the National Natural Science Foundation of China (Grants nos. 11332013, 11372295, 11002165, and 11272362), the Ministry of Education (Grant no. 313059, CHINA), Chongqing Science and Technology Commission (Grant no. cstc2013jcyjjq50003), and Fundamental Research Funds for the Central Universities (Grants nos. CDJZR12245501, CDJZR13240023, and CDJZR13240078).

\section{References}

[1] K. Ullakko, J. K. Huang, C. Kantner, R. C. O'Handley, and V. V. Kokorin, "Large magnetic-field-induced strains in $\mathrm{Ni}_{2} \mathrm{MnGa}$ single crystals," Applied Physics Letters, vol. 69, no. 13, pp. 19661968, 1996.

[2] A. Sozinov, A. A. Likhachev, N. Lanska, and K. Ullakko, "Giant magnetic-field-induced strain in NiMnGa seven-layered martensitic phase," Applied Physics Letters, vol. 80, no. 10, pp. 17461748, 2002.

[3] R. N. Couch and I. Chopra, "Experimental Characterization of NiMnGa Magnetic Shape Memory Alloy Rods Under Dynamic Magnetic Fields," in International Society for Optical Engineering: Smart Structures and Materials 2003 Active Materials: Behavior and Mechanics, Proceedings of SPIE, pp. 169-180, San Diego, Calif, USA, March 2003.

[4] R. Kainuma, Y. Imano, W. Ito et al., "Metamagnetic shape memory effect in a Heusler-type $\mathrm{Ni}_{43} \mathrm{Co}_{7} \mathrm{Mn}_{39} \mathrm{Sn}_{11}$ polycrystalline alloy," Applied Physics Letters, vol. 88, no. 19, Article ID 192513, 2006.

[5] T. Krenke, E. Duman, M. Acet et al., "Magnetic superelasticity and inverse magnetocaloric effect in Ni-Mn-In," Physical Review B: Condensed Matter and Materials Physics, vol. 75, no. 10, Article ID 104414, 2007.

[6] G. F. Dong, W. Cai, Z. Y. Gao, and J. H. Sui, "Effect of isothermal ageing on microstructure, martensitic transformation and mechanical properties of $\mathrm{Ni}_{53} \mathrm{Mn}_{23.5} \mathrm{Ga}_{18.5} \mathrm{Ti}_{5}$ ferromagnetic shape memory alloy," Scripta Materialia, vol. 58 , no. 8 , pp. $647-$ 650, 2008. 
[7] R. C. O'Handley, S. J. Murray, M. Marioni, H. Nembach, and S. M. Allen, "Phenomenology of giant magnetic-field-induced strain in ferromagnetic shape-memory materials," Journal of Applied Physics, vol. 87, no. 9, pp. 4712-4717, 2000.

[8] A. A. Likhachev and K. Ullakko, "Quantitative model of large magnetostrain effect in ferromagnetic shapell memory alloys," European Physical Journal B, vol. 14, no. 2, pp. 263-267, 2000.

[9] L. Hirsinger and C. Lexcellent, "Modelling detwinning of martensite platelets under magnetic and (or) stress actions on NiMn-Ga alloys," Journal of Magnetism and Magnetic Materials, vol. 254-255, pp. 275-277, 2003.

[10] Y. Liang, H. Kato, and M. Taya, "Model calculation of 3Dphase transformation diagram of ferromagnetic shape memory alloys," Mechanics of Materials, vol. 38, no. 5-6, pp. 564-570, 2006.

[11] N. I. Glavatska, A. A. Rudenko, I. N. Glavatskiy, and V. A. L'vov, "Statistical model of magnetostrain effect in martensite," Journal of Magnetism and Magnetic Materials, vol. 265, no. 2, pp. 142151, 2003.

[12] V. A. L'vov, S. P. Zagorodnyuk, and V. A. Chernenko, "A phenomenological theory of giant magnetoelastic response in martensite," European Physical Journal B, vol. 27, no. 1, pp. 55$62,2002$.

[13] Y. Zhu and G. Dui, "Model for field-induced reorientation strain in magnetic shape memory alloy with tensile and compressive loads," Journal of Alloys and Compounds, vol. 459, no. 1-2, pp. 55-60, 2008.

[14] Y. Pei and D. Fang, "A model for giant magnetostrain and magnetization in the martensitic phase of NiMnGa alloys," Smart Materials and Structures, vol. 16, no. 3, article 779, 2007.

[15] X. Wang and F. Li, "A kinetics model for martensite variants rearrangement in ferromagnetic shape memory alloys," Journal of Applied Physics, vol. 108, no. 11, Article ID 113921, 2010.

[16] B. Kiefer, H. E. Karaca, D. C. Lagoudas, and I. Karaman, "Characterization and modeling of the magnetic field-induced strain and work output in $\mathrm{Ni}_{2} \mathrm{MnGa}$ magnetic shape memory alloys," Journal of Magnetism and Magnetic Materials, vol. 312, no. 1, pp. 164-175, 2007.

[17] R. N. Couch, J. Sirohi, and I. Chopra, "Development of a quasistatic model of NiMnGa magnetic shape memory alloy," Journal of Intelligent Material Systems and Structures, vol. 18, no. 6, pp. 611-622, 2007.

[18] L. C. Brinson and M. S. Huang, "Simplifications and comparisons of shape memory alloy constitutive models," Journal of Intelligent Material Systems and Structures, vol. 7, no. 1, pp. 108114, 1996.

[19] X. Peng, H. Li, and W. Chen, "A comprehensive description for shape memory alloys with a two-phase mixture model incorporating the conventional theory of plasticity," Smart Materials and Structures, vol. 14, no. 2, pp. 425-433, 2005.

[20] L. C. Brinson, "One-dimensional constitutive behavior of shape memory alloys: thermomechanical derivation with non-constant material functions and redefined martensite internal variable," Journal of Intelligent Material Systems and Structures, vol. 4, no. 2, pp. 229-242, 1993.

[21] H. E. Karaca, I. Karaman, B. Basaran, Y. I. Chumlyakov, and H. J. Maier, "Magnetic field and stress induced martensite reorientation in NiMnGa ferromagnetic shape memory alloy single crystals," Acta Materialia, vol. 54, no. 1, pp. 233-245, 2006. 

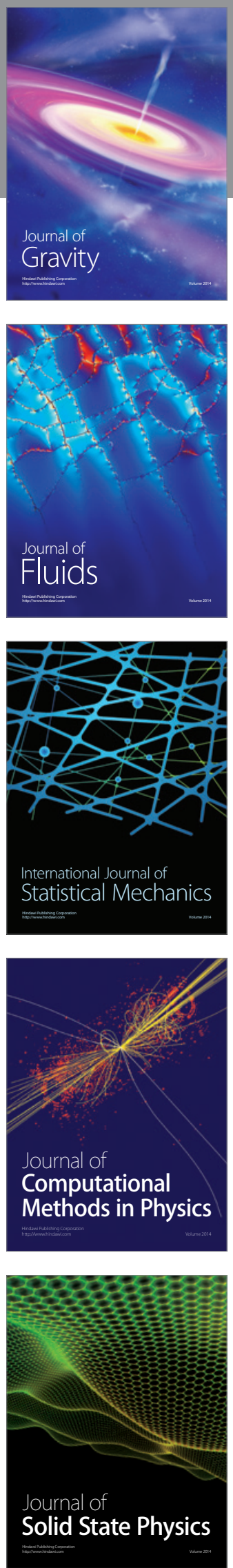

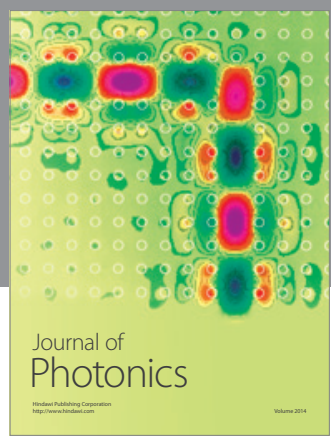

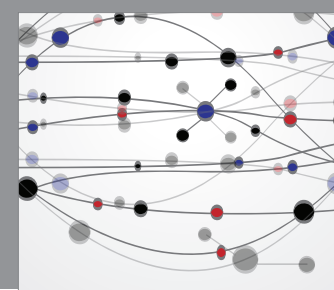

The Scientific World Journal

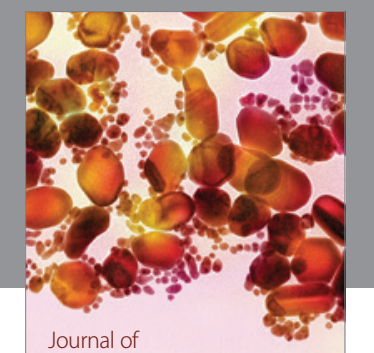

Soft Matter
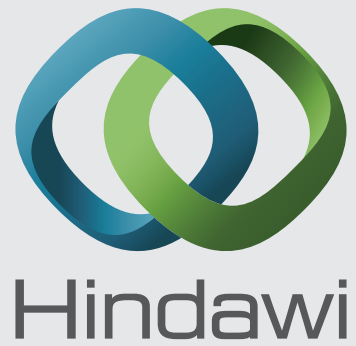

Submit your manuscripts at

http://www.hindawi.com
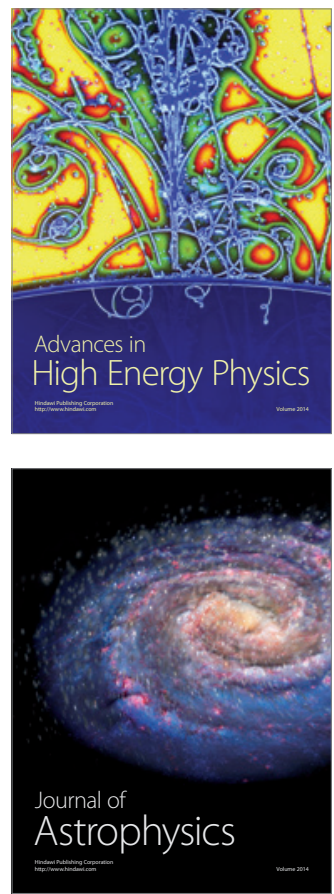
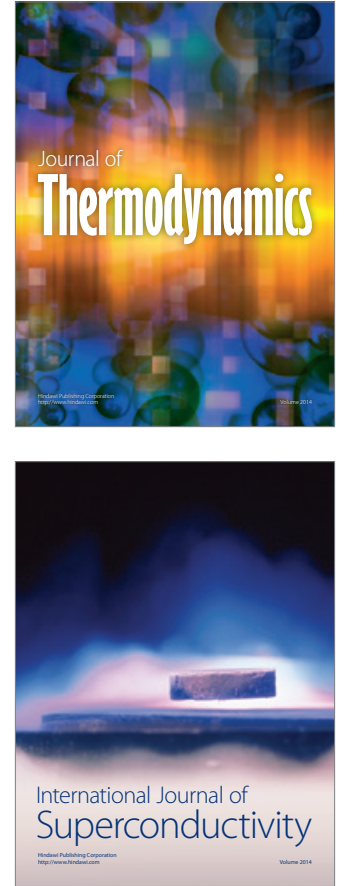
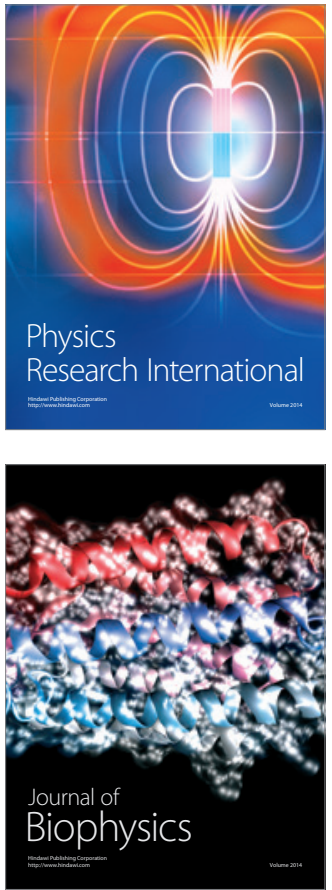
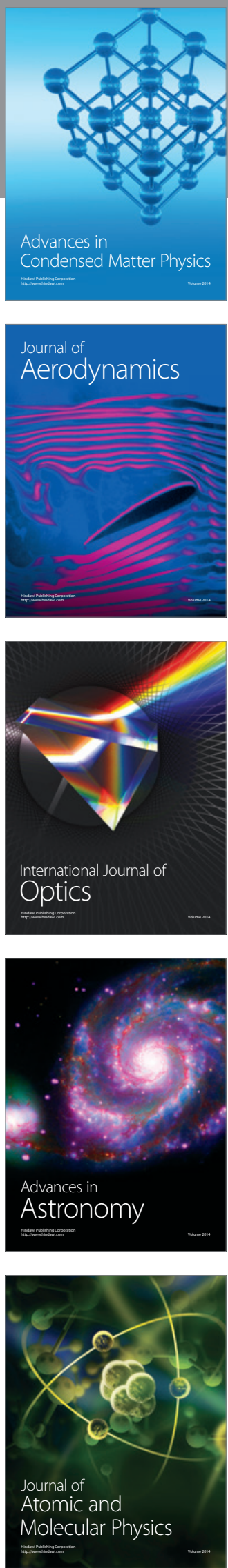